\title{
Topological Dual Systems for Spaces of Vector Measure $p$-Integrable Functions
}

\author{
P. Rueda ${ }^{1}$ and E. A. Sánchez Pérez ${ }^{2}$ \\ ${ }^{1}$ Departamento de Análisis Matemático, Universidad de Valencia, Burjassot, 46100 Valencia, Spain \\ ${ }^{2}$ Instituto Universitario de Matemática Pura y Aplicada, Universitat Politècnica de València, \\ Camino de Vera, s/n, 46022 Valencia, Spain
}

Correspondence should be addressed to E. A. Sánchez Pérez; easancpe@mat.upv.es

Received 21 April 2016; Accepted 30 May 2016

Academic Editor: Miguel Martín

Copyright (C) 2016 P. Rueda and E. A. Sánchez Pérez. This is an open access article distributed under the Creative Commons Attribution License, which permits unrestricted use, distribution, and reproduction in any medium, provided the original work is properly cited.

\begin{abstract}
We show a Dvoretzky-Rogers type theorem for the adapted version of the $q$-summing operators to the topology of the convergence of the vector valued integrals on Banach function spaces. In the pursuit of this objective we prove that the mere summability of the identity map does not guarantee that the space has to be finite dimensional, contrary to the classical case. Some local compactness assumptions on the unit balls are required. Our results open the door to new convergence theorems and tools regarding summability of series of integrable functions and approximation in function spaces, since we may find infinite dimensional spaces in which convergence of the integrals, our vector valued version of convergence in the weak topology, is equivalent to the convergence with respect to the norm. Examples and applications are also given.
\end{abstract}

\section{Introduction}

Summability in Banach spaces is one of the main topics in Functional Analysis, and results concerning the behavior of summable sequences are fundamental tool for its applications. Comparison between norm and weak absolutely summable series is at the origin of some classical problems in the theory of Banach spaces, and it was the starting point of the theory of $p$-summing operators. In this paper we are interested in providing new elements for the analysis of summability in the case of Banach function spaces by using a vector valued duality that is provided by the vector measure integration theory on spaces $L^{p}(m)$ of integrable functions with respect to a vector measure $m$. These spaces represent, in fact, all order continuous $p$-convex Banach lattices with weak unit. This theory supplies a distinguished element, the vector valued integral, for the study of summability in Banach spaces of measurable functions. It is well known that $f g \in L^{1}(m)$ whenever $f \in L^{p}(m)$ and $g \in L^{p^{\prime}}(m), 1 / p+1 / p^{\prime}=1$. In this case, the integral $\int \mathrm{fg} d m$ determines a vector valued bilinear map that yields to duality: the vector valued duality between $L^{p}(m)$ and $L^{p^{\prime}}(m)($ see $[1,2])$.
This vector valued duality is the framework to study natural topologies on spaces of integrable functions with respect to a vector measure, as the topology $\tau_{m}$ generated by the seminorms $\gamma_{g}(f):=\left\|\int f g d m\right\|$ and $f \in L^{p}(m)$, when varying $g \in L^{p^{\prime}}(m)$. This new vector valued point of view was first taken into consideration in the study of convergence of sequences: the relation between the convergence of sequences in spaces of vector measure integrable functions and the convergence of the corresponding vector valued integrals has been treated since the seventies (see, e.g., $[3,4]$, $[5$, Section 6], [6], and the references therein). In this paper we are interested in the summability of sequences in $L^{p}(m)$ spaces induced by the vector valued duality, that is, when the role played by the weak topology is assumed by the topology $\tau_{m}$. It is worth mentioning that the $p$-convexification $L^{p}(m)$ $(p \geq 1)$ of the space $L^{1}(m)$ of a vector measure $m$ was introduced as a tool for analyzing summability (see [1]), trying to bring together vector valued integration and the theory of $p$-summing operators in Banach spaces (see also $[7,8])$.

The classical Dvoretzky-Rogers theorem can be stated as follows: the identity map in a Banach space $E$ is absolutely 
$q$-summing for some $1 \leq q<\infty$, if and only if $E$ is finite dimensional. This paper is devoted to proving an extension for Banach function spaces of this result. In our context, the usual scalar duality is replaced by the vector valued duality given by a vector measure and the role of the weak topology in the Banach space is assumed by the topology $\tau_{m}$. In order to develop our study, we analyze some properties of the $\left(q, P^{m}\right)$-summing operators that map $\tau_{m}$ summable sequences to norm summable sequences. Our main result shows the necessity of adding some topological requirements on local compactness to characterize finite dimensional spaces in terms of the $\left(q, P^{m}\right)$-summability of the identity map. The last section shows an application to the study of subspaces of $L^{p}(m)$ that are fixed by the integration operator. As a consequence of our Dvoretzky-Rogers type theorem, we prove that, under the local compactness hypotheses, only finite dimensional subspaces can be fixed by the integration map.

\section{Preliminaries}

We use standard Banach space notation. Let $1 \leq p \leq \infty$. Then we write $p^{\prime}$ for the extended real number satisfying $1 / p+1 / p^{\prime}=1$. We follow the definition of Banach function space over a finite measure $\mu$ given in [9, Def. 1.b.17, p. 28]. Throughout the paper $X(\mu)$ will denote an infinite dimensional Banach function space over $\mu$; that is, $X(\mu)$ is a Banach lattice of $\mu$, a.e., equal classes of $\mu$-integrable functions with a lattice norm and the $\mu$ a.e. order satisfying $L^{\infty}(\mu) \subseteq X(\mu) \subseteq L^{1}(\mu)$. We will also assume that $X(\mu)$ is order continuous; that is, for each decreasing sequence $f_{n} \downarrow 0$ in $X(\mu), \lim _{n}\left\|f_{n}\right\|_{X(\mu)}=0$.

Let $X$ be a real Banach space and let $(\Omega, \Sigma)$ be a measurable space. If $m: \Sigma \rightarrow X$ is a countably additive vector measure, we write $\mathscr{R}(m)$ for its range. The variation $|m|$ of $m$ is given by $|m|(A):=\sup _{B_{i} \in \pi} \sum_{i=1}^{n}\left\|m\left(B_{i}\right)\right\|$, where the supremum is computed over all finite measurable partitions $\pi$ of $A \in \Sigma$. $\|m\|$ is the semivariation of $m$; that is, $\|m\|(A):=$ $\sup _{x^{*} \in B_{X^{*}}}\left|\left\langle m, x^{*}\right\rangle\right|(A), A \in \Sigma$, where $\left\langle m, x^{*}\right\rangle$ is the scalar measure given by $\left\langle m, x^{*}\right\rangle(A):=\left\langle m(A), x^{*}\right\rangle$. The Rybakov Theorem (see $\left[10, \mathrm{Ch}\right.$. IX]) establishes that there exists $x^{*} \in$ $X^{*}$ such that $m$ is absolutely continuous with respect to a socalled Rybakov measure $\left|\left\langle m, x^{*}\right\rangle\right|$ that means that $m(A)=0$ whenever $\left|\left\langle m(A), x^{*}\right\rangle\right|=0$. For $1 \leq p<\infty$, a (real) measurable function $f$ is said to be $p$-integrable with respect to $m$ if $|f|^{p}$ is integrable with respect to all measures $\left|\left\langle m, x^{*}\right\rangle\right|$ and for each $A \in \Sigma$ there exists an element $\int_{A}|f|^{p} d m \in X$ such that $\left\langle\int_{A}|f|^{p} d m, x^{*}\right\rangle=\int_{A}|f|^{p} d\left\langle m, x^{*}\right\rangle, x^{*} \in X^{*}$.

The space $L^{p}(m), 1 \leq p<\infty$, is defined to be the Banach lattice of all ( $\mu$-equivalence classes of) measurable real functions defined on $\Omega$ that are $p$-integrable with respect to $m$ when the a.e. order and the norm

$$
\begin{aligned}
\|f\|_{L^{p}(m)}:=\left(\sup _{x^{*} \in B_{X^{*}}} \int|f|^{p} d\left|\left\langle m, x^{*}\right\rangle\right|\right)^{1 / p}, & \\
& f \in L^{p}(m),
\end{aligned}
$$

are considered. It is an order continuous $p$-convex Banach function space over any Rybakov measure $\eta$ for $m$ (see [1, Proposition 5]; see also [11] and [6, Ch. 3] for more information on these spaces). For the case $p=\infty, L^{\infty}(m)$ is defined as $L^{\infty}(\eta)$. A relevant fact is that, for each $1 \leq p \leq \infty$, $L^{p}(m) \cdot L^{p^{\prime}}(m) \subseteq L^{1}(m)$ (see [6, Prop. 3.43] and [1, Sec. 3]; see also [11]). Moreover, for each $f \in L^{p}(m)$

$$
\|f\|_{L^{p}(m)}=\sup _{g \in B_{L^{p^{\prime}}(m)}}\left\|\int f g d m\right\| .
$$

These relations allow defining the so-called vector measure duality by using the integration operator $I_{m}: L^{1}(m) \rightarrow X$, which is given by

$$
I_{m}(f)=\int_{\Omega} f d m, \quad f \in L^{1}(m) .
$$

We will use the symbol $\int f d m$ instead of $\int_{\Omega} f d m$ throughout the paper. Relevant information on the properties of $I_{m}$ can be found in [12-14] and [6, Ch. 3] and the references therein. Since for all $p>1$ the inclusion $L^{p}(m) \subseteq L^{1}(m)$ always holds, the integration map can be defined also as an operator $I_{m}: L^{p}(m) \rightarrow X$; we use the same symbol $I_{m}$ in this case for this operator. It must be said that the spaces $L^{p}(m)$ represent in fact the class of all order continuous $p$-convex Banach lattices with a weak unit (see [11, Prop. 2.4] or [6, Prop. 3.30]) that means that our results can be applied to a broad class of Banach spaces.

As we said in Introduction, duality and vector valued duality for the spaces $L^{p}(m)$ are fundamental tools in this paper. Regarding duality, fix a Rybakov measure $\mu$ for $m$. Due to the order continuity of $L^{p}(m)$, its dual space $L^{p}(m)^{*}$ $(1 \leq p<\infty)$ allows an easy description; it coincides with its Köthe dual (or associate space) $\left(L^{p}(m)\right)^{\prime}$; that is, $L^{p}(m)^{*}=$ $\left(L^{p}(m)\right)^{\prime}=\left\{\varphi_{g}: g \in \mathscr{H}\right\}$, where

$$
\begin{aligned}
\mathscr{H} & :=\left\{g: \Omega \longrightarrow \mathbb{R} \Sigma \text {-measurable }: f g \in L^{1}(\mu) \forall f\right. \\
& \left.\in L^{p}(m)\right\}
\end{aligned}
$$

and the duality is given by $\left\langle\varphi_{g}, f\right\rangle=\int_{\Omega} f g d \mu$. Information about a precise description of $\left(L^{p}(m)\right)^{\prime}$ can be found in $[2,7,15-17]$. It must be said here that $\left(L^{p}(m)\right)^{\prime}$ and $L^{p^{\prime}}(m)$ coincide only in very special situations, for instance, for $m$ being a scalar measure. We will write $\tau_{w}$ for the weak topology on $L^{p}(m)$.

Regarding vector valued duality relations between $L^{p}(m)$ spaces, $1 \leq p \leq \infty$, the integration map defines the continuous bilinear map

$$
B_{m}: L^{p}(m) \times L^{p^{\prime}}(m) \longrightarrow X
$$

given by $B_{m}(f, g):=\int f g d m, f \in L^{p}(m), g \in L^{p^{\prime}}(m)$. Note that $B_{m}$ is both sides norming for $L^{p}(m)$ and $L^{p^{\prime}}(m)$; that is, for every $f \in L^{p}(m),\|f\|_{L^{p}(m)}=\sup _{g \in B_{L^{p^{\prime}}(m)}}\left\|\int f g d m\right\|$, and the same happens dually for the case of functions $g \in L^{p^{\prime}}(m)$. 
In this paper we will consider the topology $\tau_{m}$ of pointwise convergence of the integrals that is the locally convex topology defined by the seminorms $\gamma_{g}(f):=\left\|\int f g d m\right\|_{X}$, $f \in L^{p}(m), g \in L^{p^{\prime}}(m)$. The topology $\tau_{w, m}$ of pointwise weak convergence of the integrals is defined by the seminorms $\gamma_{g, x^{*}}(f):=\left\langle\int f g d m, x^{*}\right\rangle, f \in L^{p}(m), g \in L^{p^{\prime}}(m)$, and $x^{*} \in X^{*}$. It is also a locally convex topology on $L^{p}(m)$. It is easy to see that the norm topology is finer than all the others, and $\tau_{m}$ and $\tau_{w}$ are finer than $\tau_{w, m}$, although $\tau_{m}$ and $\tau_{w}$ are not comparable in general. An exhaustive analysis of the $\tau_{m}$ topology has been done recently and can be found in [18] (see also the references therein). The reader can find more information about it in $[1,6,7,11,16,19]$. The following result establishes the basic relations between the quoted topologies.

Proposition 1 (See Proposition 1 in [18]). Let $1 \leq p \leq \infty$. If $B_{L^{p}(m)}$ is $\tau_{m}$-compact then $\tau_{w, m}$ and $\tau_{m}$ coincide on bounded subsets of $L^{p}(m)$. Moreover, if $p>1$ and $B_{L^{p}(m)}$ is $\tau_{m}$-compact then the weak topology and $\tau_{m}$ coincide on bounded subsets of $L^{p}(m)$. Consequently, if $p>1, B_{L^{p}(m)}$ is $\tau_{m^{-} \text {-compact if and only }}$ if $\left(L^{p}(m),\|\cdot\|_{L^{p}(m)}\right)$ is reflexive and the weak topology and $\tau_{m}$ coincide on $B_{L^{p}(m)}$.

In this paper we will make a local use of the duality defined by the integration bilinear map $B_{m}$. For $1 \leq p \leq \infty$ consider a subspace $P \subseteq L^{p}(m)$. We say that a subspace $R \subseteq$ $L^{p^{\prime}}(m)$ is an $m$-dual for $P$ if $R$ is $m$-norming for $P$; that is, the function $f \leadsto \sup _{g \in B_{R}}\left\|\int f g d m\right\|$ gives an equivalent norm for $P$. We write $P^{m}$ for such a space $R$. In the same way, we say that a subspace $P^{m m}$ of $L^{P}(m)$ is $m$-bidual of $P$ (with respect to $m$-dual $\left.P^{m}\right)$ if $P \subseteq P^{m m}$ and $P^{m m}$ is $m$-norming for $P^{m}$. Notice that the inclusion $P \subseteq P^{m m}$ is not necessary for $P^{m m}$ to be $m$ norming for $P^{m}$. For instance, if $X(\mu)$ is an order continuous Banach function space and $m: \Sigma \rightarrow X(\mu)$ is the vector measure given by $m(A):=\chi_{A}, A \in \Sigma$, then for $P=L^{p}(m)$ the space $P^{m}$ generated by the function $\chi_{\Omega}$ in $L^{p^{\prime}}(m)$ is $m$ norming for $P$, and also the space $P^{m m}$ generated by $\chi_{\Omega}$ in $L^{p}(m)$ is $m$-norming for $P^{m}$. However, $P$ is not included in $P^{m m}$. But note also that given $P, P^{m}$, and $P^{m m}$ being norming, it can always be assumed that $P \subseteq P^{m m}$ just by defining the new $P^{m m}$ as the subspace of $L^{p}(m)$ generated by $P \cup P^{m m}$. We will use this example later.

We say that a triple $\left(P, P^{m}, P^{m m}\right)$ of $m$-dual spaces as above is an $m$-dual system. We can define the topology $\tau_{m}\left(P^{m}\right)$ over $P$ as the one induced by all the seminorms $f \leadsto$ $\left\|\int f g d m\right\|, g \in P^{m}$, and $\tau\left(P^{m m}\right)$ the topology for $P^{m}$ given by the seminorms $g \leadsto\left\|\int f g d m\right\|, f \in P^{m m}$. A quick look at the proof of Proposition 1 in [18] shows that a local version of this result is also true, that is, a version of this result writing $P$ instead of $L^{p}(m)$ and $\tau_{m}\left(P^{m}\right)$ instead of $\tau_{m}$, where $P^{m}$ is an $m$-dual space.

Let us show some examples. A natural $m$-dual space of $L^{p}(m)$ is $L^{p^{\prime}}(m)$; in this case, we write simply $\tau_{m}$ for the topology $\tau_{m}\left(L^{p^{\prime}}(m)\right)$. However, an $m$-dual space may be very small. For instance, if the integration map $I_{m}: L^{1}(m) \rightarrow X$ is isomorphism, then the subspace generated by $\chi_{\Omega} \in L^{\infty}(m)$ is $m$-dual for $L^{1}(m)$. Obviously, for every subspace $P \subseteq L^{p}(m)$, $L^{p^{\prime}}(m)$ is $m$-dual for $P$.

Let us finish this section by defining a fundamental class of operators related to the summability of sequences with respect to the $\tau_{m}$-topology. It generalizes the class considered in Lemma 16 of [1] and in [7, Section 4.2]. Theorem 17 in [1] provides a Pietsch type domination/factorization theorem for this family of operators. The local version of this result becomes the main tool for the proof of our results.

Definition 2. Let $1 \leq p, q<\infty, R$ be a subspace of $L^{p^{\prime}}(m)$ and $P$ a Banach subspace of $L^{p}(m)$. Let $E$ be a Banach space. An operator $T: P \rightarrow E$ is $(q, R)$-summing if there is a constant $K$ such that, for any finite set of functions $f_{1}, \ldots, f_{n} \in P$,

$$
\left(\sum_{i=1}^{n}\left\|T\left(f_{i}\right)\right\|^{q}\right)^{1 / q} \leq K \sup _{g \in B_{R}}\left(\sum_{i=1}^{n}\left\|\int f_{i} g d m\right\|^{q}\right)^{1 / q} .
$$

Of course, the integration map $I_{m}: P \rightarrow X$ is always $\left(q, L^{p^{\prime}}(m)\right)$-summing for all $1 \leq p, q<\infty$. Indeed, if $f_{1}, \ldots, f_{n} \in L^{p}(m)$, then

$$
\begin{aligned}
\sum_{i=1}^{n}\left\|I_{m}\left(f_{i}\right)\right\|^{q} \leq & \|m\|(\Omega)^{q / p^{\prime}} \\
& \cdot \sup _{h \in B_{L^{p^{\prime}}(m)}}\left(\sum_{i=1}^{n}\left\|\int f_{i} h d m\right\|^{q}\right) .
\end{aligned}
$$

\section{The Dvoretzky-Rogers Theorem for the $m$-Summability}

Throughout this section, $1 \leq p \leq \infty, E$ and $X$ are Banach spaces, $m$ is an $X$-valued vector measure, $P$ is a subspace of $L^{P}(m)$, and $\left(P, P^{m}, P^{m m}\right)$ is an $m$-dual system. We will consider the following sequential properties associated with compactness with respect to the $\tau_{m}$-topology.

Definition 3. An operator $T: E \rightarrow P$ is $\tau_{m}\left(P^{m}\right)$ sequentially compact if every bounded sequence $\left(x_{n}\right)_{n}$ in $E$ has a subsequence $\left(x_{n_{k}}\right)_{k}$ such that $\left(\int T\left(x_{n_{k}}\right) g d m\right)_{k}$ is a Cauchy sequence for each $g \in P^{m}$.

Definition 4. An operator $T: P \rightarrow E$ is $\tau_{m}\left(P^{m}\right)$-sequentially completely continuous if $\lim _{n}\left\|T\left(f_{n}\right)\right\|=0$ whenever $\left(f_{n}\right)_{n}$ is a bounded sequence such that $\lim _{n}\left\|\int h f_{n} d m\right\|=0$ for every $h \in P^{m}$

If we assume that $\chi_{\Omega} \in P^{m}$ (we can always make $P^{m}$ big enough to have it), then $I_{m}: P \rightarrow X$ is $\tau_{m}\left(P^{m}\right)$-sequentially continuous. In the classical summing operators theory it is well known that any summing operator is weakly compact. However, not every $\left(q, P^{m}\right)$-summing operator is $\tau_{m}\left(P^{m}\right)$ sequentially compact. For instance, given a Banach function space $X(\mu)$, define $m(A):=\chi_{A}, A \in \Sigma$. Then $I_{m}: L^{1}(m) \rightarrow$ $X(\mu)$ is an isomorphism which is $\left(q, L^{\infty}(m)\right)$-summing but it is not $\tau_{m}\left(L^{\infty}(m)\right)$-sequentially compact in general as in this case the norm topology and the $\tau_{m}=\tau_{m}\left(L^{\infty}(m)\right)$ 
topology coincide. Then $B_{L^{1}(m)}$ is not $\tau_{m}$-compact unless $L^{1}(m)$ is finite dimensional. Let us see that, under some compactness assumptions, the $\left(q, P^{m}\right)$-summing operators behave similarly as absolutely summing operators. We need first an easy lemma.

Lemma 5. Let $1 \leq p \leq \infty$ and let $P$ be a subspace of $L^{p}(m)$ and $P^{m}$ an $m$-norming subspace for $P$. Consider a Banach space valued $\left(r, P^{m}\right)$-summing operator $T: P \rightarrow E$. Then $T$ is $\left(q, P^{m}\right)$-summing for each $1 \leq r \leq q<\infty$.

Proof. Let $s$ be such that $1 / q+1 / s=1 / r$. Take a finite set of functions $f_{1}, \ldots, f_{n} \in P$. Then

$$
\begin{aligned}
& \left(\sum_{i=1}^{n}\left\|T\left(f_{i}\right)\right\|^{q}\right)^{1 / q}=\sup _{\left(\lambda_{i}\right)_{i=1}^{n} \in B_{\ell^{s}}}\left(\sum_{i=1}^{n}\left|\lambda_{i}\right|^{r}\left\|T\left(f_{i}\right)\right\|^{r}\right)^{1 / r} \\
& \leq \min _{\left(\lambda_{i}\right)_{i=1}^{n} \in B_{\ell^{s}}}\left(\sum_{i=1}^{n}\left\|T\left(\lambda_{i} f_{i}\right)\right\|^{r}\right)^{1 / r} \\
& \leq \sup _{\left(\lambda_{i}\right)_{i=1}^{n} \in B_{\ell^{s}}} K \sup _{g \in B_{P^{m}}}\left(\sum_{i=1}^{n}\left|\lambda_{i}\right|^{r}\left\|\int f_{i} g d m\right\|^{r}\right)^{1 / r} \\
& \leq K \sup _{g \in B_{P^{m}}}\left(\sum_{i=1}^{n}\left\|\int f_{i} g d m\right\|^{q}\right)^{1 / q}
\end{aligned}
$$

where $K$ is the constant associated with the $\left(r, P^{m}\right)$ summability of $T$.

Theorem 6. Let $1 \leq q<\infty$. Let $T: P \rightarrow$ E be a $\left(q, P^{m}\right)$ summing operator. The following statements hold.

(i) If $B_{P^{m}}$ is $\tau_{m}\left(P^{m m}\right)$-compact then $T$ is $\tau_{m}\left(P^{m}\right)$-sequentially completely continuous.

(ii) If $B_{P}$ is $\tau_{m}\left(P^{m}\right)$-compact and $B_{P^{m}}$ is $\tau_{m}\left(P^{m m}\right)$-compact then $T$ is completely continuous.

(iii) Finally, if $B_{P^{m}}$ is $\tau_{m}\left(P^{m m}\right)$-compact and $X$ is reflexive, then $T$ is also weakly compact.

Proof. (i) We have that $T$ satisfies that, for every finite set $f_{1}, \ldots, f_{n} \in P$,

$$
\sum_{i=1}^{n}\left\|T\left(f_{i}\right)\right\|^{q} \leq K^{q} \sup _{g \in B_{P} m} \sum_{i=1}^{n}\left\|\int f_{i} g d m\right\|^{q} .
$$

Taking into account that $\left(P, P^{m}, P^{m m}\right)$ is an $m$-dual system, it can be shown as in the case of Pietsch's Domination Theorem for $q$-summing operators (see Lemma 16 in [1] and make the obvious modifications) that there is measure $\eta$ on the compact space $\left(B_{P^{m}}, \tau_{m}\left(P^{m m}\right)\right)$ such that

$$
\|T(f)\| \leq K\left(\int_{B_{P^{m}}}\left\|\int f g d m\right\|^{q} d \eta(g)\right)^{1 / q}, \quad f \in P .
$$

This easily gives that $T$ factorizes through the following scheme (see Theorem 17 in [1]):

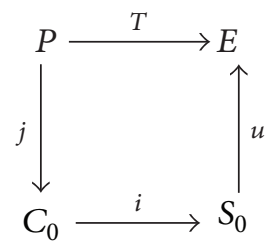

where $C_{0}$ is the subspace of $C\left(B_{P^{m}}, X\right)$ given by the functions $g \leadsto \int f g d m \in X, j$ is the isomorphism given by the identification of a function $f$ with the corresponding vector valued function in $C_{0}, S_{0}$ is the closure of the image of $C_{0}$ by the natural inclusion/quotient map

$$
C\left(B_{P^{m}}, X\right) \longrightarrow L^{q}\left(B_{P^{m}}, \eta, X\right),
$$

where $\eta$ is a Radon probability measure on $B_{P^{m}}$, and $u$ is the map that closes the diagram. Using this scheme, an argument based on the Dominated Convergence Theorem gives the result. Let $\left(h_{n}\right)_{n}$ be a bounded sequence in $P$ such that the sequence of integrals $\left(\left\|\int h_{n} g d m\right\|\right)_{n}$ is null for every $g \in P^{m}$. It is enough to prove that the sequence of functions $g \leadsto$ $\int h_{n} g d m \in X$ satisfies $\lim _{n} \int_{g \in B_{p} m}\left\|\int h_{n} g d m\right\|^{q} d \eta(g)=0$. For each $n$, the function $\varphi_{n}(\cdot):=\left\|\int h_{n} \cdot d m\right\|$ belongs to the space $C\left(B_{P^{m}}\right)$ of scalar continuous functions defined on the compact set $\left(B_{P^{m}}, \tau_{m}\left(P^{m m}\right)\right)$. Since there is a constant $K>0$ such that $\varphi_{n}(g) \leq K \chi_{B_{P} m}(g)$ for all $g \in B_{P^{m}}$ and $n$, we can apply the Lebesgue Dominated Convergence Theorem to obtain that

$$
\begin{aligned}
& \lim _{n} \int_{B_{P^{m}}}\left\|\int h_{n} g d m\right\|^{q} d \eta(g)=\lim _{n} \int_{B_{P^{m}}} \varphi_{n}^{q}(g) d \eta(g) \\
& \quad=0 .
\end{aligned}
$$

Therefore, using the factorization we obtain that $\lim _{n}\left\|T\left(h_{n}\right)\right\|=0$ and so $T$ is $\tau_{m}\left(P^{m}\right)$-sequentially completely continuous.

(ii) Let $\left(f_{n}\right)_{n}$ be a weakly null sequence in $P$. Since $\left(B_{P^{m}}, \tau_{m}\left(P^{m m}\right)\right)$ is compact, using the factorization given in (i) and taking into account that each continuous operator is weak-to-weak continuous we get that for each element $\delta_{g} \otimes x^{\prime} \in\left(C\left(B_{P^{m}}, X\right)\right)^{\prime}, g \in P^{m}, x^{\prime} \in X^{\prime}$, we have that

$$
\lim _{n}\left\langle\int f_{n} \cdot d m, \delta_{g} \otimes x^{\prime}\right\rangle=\lim _{n}\left\langle\int f_{n} g d m, x^{\prime}\right\rangle=0 .
$$

Due to an easy adaptation of Proposition 1, since we are assuming that $\left(B_{P}, \tau_{m}\left(P^{m}\right)\right)$ is compact, the topologies $\tau_{w, m}\left(P^{m}\right)$, generated by the seminorms $\gamma_{x^{*}, g}(f)$ := $\left\langle\int f g d m, x^{*}\right\rangle$ when varying $x^{*} \in X^{\prime}$ and $g \in P^{m}$, and $\tau_{m}\left(P^{m}\right)$ coincide on $B_{P}$.

Consequently for each $g \in P^{m}, \lim _{n}\left\|\int f_{n} g d m\right\|=0$. Using the domination in (i), we obtain the result on the complete continuity.

(iii) Finally, by Lemma 5 if $T$ is $\left(q, P^{m}\right)$-summing it is $\left(s, P^{m}\right)$-summing for $q<s<\infty$, and so the reflexivity of $X$ implies the reflexivity of $L^{s}\left(B_{P^{m}}, \eta, X\right)$. Thus, the factorization of $T$ through a subspace of $L^{s}\left(B_{P^{m}}, \eta, X\right)$ gives that $T$ is weakly compact. 
The following result is a direct consequence of statements (ii) and (iii) of Theorem 6.

Corollary 7. Suppose that $m$ is an $X$-valued vector measure and $X$ is reflexive. Let $T: P \rightarrow P$ be $a\left(q, P^{m}\right)$-summing operator, and suppose that $B_{P}$ is $\tau_{m}\left(P^{m}\right)$-compact and $B_{P^{m}}$ is $\tau_{m}\left(P^{m m}\right)$-compact. Then $T \circ T$ is compact.

In particular, if $T: P \rightarrow E$ is isomorphism in Corollary 7, we obtain that $P$ has to be finite dimensional.

Example 8. Let us show an example of a proper infinite dimensional subspace of a space $L^{2}(m)$ with an $m$-dual system in which $P, P^{m}$, and $P^{m m}$ coincide. However, the identity map is not $\left(q, P^{m}\right)$-summing for any $1 \leq q<$ $\infty$. Take an infinite nontrivial measurable partition $\left\{A_{i}\right\}_{i=1}^{\infty}$ of the Lebesgue space $([0,1], \mathscr{B}, \mu)$, and define the vector measure $m: \mathscr{B} \rightarrow \ell^{2}$ given by $m(B):=\sum_{i=1}^{\infty} \mu\left(A_{i} \cap B\right) e_{i}$, where $\left\{e_{i}: i=1, \ldots\right\}$ is the canonical basis of $\ell^{2}$ and $B \in \mathscr{B}$ (see Example 10 in [20]). Consider the (infinite dimensional closed) subspace $P$ of $L^{2}(m)$ generated by the functions $\chi_{A_{i}} / \mu\left(A_{i}\right)^{1 / 2}, i \in \mathbb{N}$. A direct calculation shows that, for each $f=\sum_{i=1}^{\infty} \lambda_{i} \chi_{A_{i}} / \mu\left(A_{i}\right)^{1 / 2} \in P$,

$$
\|f\|_{L^{2}(m)}=\left(\sum_{i=1}^{\infty}\left|\lambda_{i}\right|^{4}\right)^{1 / 4}
$$

and so $P$ is isometric to $\ell^{4}$ (see Proposition 11 in [20]). We can define the $m$-dual space $P^{m} \subseteq L^{2}(m)$ and the $m$-bidual space $P^{m m}$ as $P^{m}=P^{m m}=P$. It is clear that $P^{m}$ norms $P$ and $P^{m m}$ norms $P^{m}$. However, the identity map is not $\left(q, P^{m}\right)$-summing for any $1 \leq q<\infty$. In order to see this, consider the sequence of functions $\left(\chi_{A_{i}} / \mu\left(A_{i}\right)^{1 / 2}\right)_{i=1}^{\infty}$. Then, if $1 \leq q<\infty$, for each $k \in \mathbb{N}$ we get

$$
\sum_{i=1}^{k}\left\|\frac{\chi_{A_{i}}}{\mu\left(A_{i}\right)^{1 / 2}}\right\|_{L^{2}(m)}^{q}=k,
$$

but

$$
\begin{gathered}
\sup _{g \in B_{P^{m}}} \sum_{i=1}^{k}\left\|\int \frac{\chi_{A_{i}}}{\mu\left(A_{i}\right)^{1 / 2}} g d m\right\|_{\ell^{2}}^{q}=\sup _{\left(\tau_{i}\right)_{i=1}^{\infty} \in B_{\ell^{4}}} \sum_{i=1}^{k}\left|\tau_{i}\right|^{q} \\
\leq \sup _{\left(\tau_{i}\right)_{i=1}^{\infty} \in B_{\ell^{4}}}\left(\sum_{i=1}^{k}\left|\tau_{i}\right|^{4 q}\right)^{1 / 4} \cdot k^{3 / 4} \leq k^{3 / 4} .
\end{gathered}
$$

This gives a contradiction and shows that the identity map cannot be $\left(q, P^{m}\right)$-summing for any $1 \leq q<\infty$. Note that the range of $m$ is relatively compact, since it can be included in the convex hull of a null sequence of $\ell^{2}$. Corollary 8 in [18] establishes that for a reflexive and separable space $L^{2}(m)$ (our space satisfies both requirements) relative compactness of the range of $m$ implies compactness of $\left(B_{L^{2}(m)}, \tau_{m}\right) . B_{P}$ is $\tau_{m}$-closed, since by Proposition 1, $\tau_{m}$ is finer than the weak topology on $L^{2}(m)$. This gives compactness of $\left(B_{P}, \tau_{m}\left(P^{m}\right)\right)$, since the topology $\tau_{m}\left(P^{m}\right)$ is weaker than the topology $\tau_{m}$ on
$B_{P}$, and so compactness of $\left(B_{P^{m}}, \tau_{m}\left(P^{m m}\right)\right)$. The topological requirements of Corollary 7 are then satisfied and $P$ is reflexive, but obviously the identity map is not compact. Since $\ell^{4}$ is not a Schur space, the identity map is not completely continuous. This shows that the summability condition in Theorem 6(ii) and in Corollary 7 cannot be dropped.

The following is our main result and gives a vector measure version of the Dvoretzky-Rogers theorem.

Theorem 9. Let $E$ be a Banach space, $P$ a subspace of $L^{p}(m)$, and $T: P \rightarrow E$ isomorphism. The following statements are equivalent.

(i) There is an $m$-dual system $\left(P, P^{m}, P^{m m}\right)$ such that $B_{P}$ is $\tau_{m}\left(P^{m}\right)$-sequentially compact, $B_{P^{m}}$ is $\tau_{m}\left(P^{m m}\right)$ compact, and $T$ is $\left(q, P^{m}\right)$-summing for some and then, for all, $1 \leq q<\infty$.

(ii) $P$ has finite dimension.

Proof. (i) $\Rightarrow$ (ii) Assume that $T$ is $\left(q, P^{m}\right)$-summing for fixed $1 \leq q<\infty$. Let us show that the composition $T \circ T^{-1}$ is compact. As a consequence of Theorem 6(i), we know that $T$ is $\tau_{m}\left(P^{m}\right)$-sequentially completely continuous. Since $B_{P}$ is $\tau_{m}\left(P^{m}\right)$-sequentially compact, $T^{-1}: T(E) \rightarrow P$ is $\tau_{m}\left(P^{m}\right)$ sequentially compact. Then the identity map $T \circ T^{-1}: P \rightarrow P$ is compact, and so $P$ has finite dimension.

(ii) $\Rightarrow$ (i) Since $P$ is finite dimensional, we have that $\left(S_{P}, \|\right.$. $\left.\|_{L^{p}(m)}\right)$ is compact. The norm topology is finer than $\tau_{m}$, and so the unit sphere $\left(S_{P}, \tau_{m}\right)$ is compact too. For each element $f \in S_{P}$, take a norm one function $g_{f} \in L^{p^{\prime}}(m)$ that satisfies that $1 / 2 \leq\left\|\int f g_{f} d m\right\| \leq 1$. Consider the $\tau_{m}$-open covering of $S_{P}$ given by the sets

$$
\left\{h \in L^{p}(m):\left\|\int(h-f) g_{f} d m\right\|<\frac{1}{4}, f \in S_{P}\right\} .
$$

There is finite subcovering given by a finite set $\mathscr{C}=\left\{g_{f_{i}}: i=\right.$ $1, \ldots, n\}$ of such functions $g_{f}$. Then we define $P^{m}$ to be the subspace generated by $\mathscr{C}$. Note that for each $f \in S_{P}$ there is an index $i \in\{1, \ldots, n\}$ such that $\left\|\int\left(f_{i}-f\right) g_{f_{i}} d m\right\|<1 / 4$ and so

$$
\begin{aligned}
\frac{1}{2} & \leq\left\|\int f_{i} g_{f_{i}} d m\right\| \\
& \leq\left\|\int f g_{f_{i}} d m\right\|+\left\|\int\left(f_{i}-f\right) g_{f_{i}} d m\right\| \\
& \leq\left\|\int f g_{f_{i}} d m\right\|+\frac{1}{4} \leq \sup _{g \in B_{P^{m}}}\left\|\int f g d m\right\|+\frac{1}{4} \\
& \leq\|f\|_{L^{p}(m)} \cdot \sup _{g \in B_{P^{m}}}\|g\|_{L^{p^{\prime}(m)}}+\frac{1}{4} \leq 1+\frac{1}{4} .
\end{aligned}
$$

Consequently, for each $f \in P$,

$$
\frac{1}{4}\|f\|_{L^{p}(m)} \leq \sup _{g \in B_{P^{m}}}\left\|\int f g d m\right\| \leq\|f\|_{L^{p}(m)} .
$$


Therefore, the space $P^{m}$ is $m$-norming for $P$ and $B_{P}$ is $\tau_{m}\left(P^{m}\right)$ sequentially compact since the norm topology and $\tau_{m}\left(P^{m}\right)$ coincide in the finite dimensional space $P$.

Note that we can also define a finite dimensional subspace $P^{m m}$ containing $P$ that is $m$-norming for $P^{m}$ following the same procedure in the definition of $P^{m}$. The finite dimension of $P^{m}$ proves also that $B_{P^{m}}$ is $\tau_{m}\left(P^{m m}\right)$-compact.

Finally, let us see that $T$ is $\left(q, P^{m}\right)$-summing for all $1 \leq q$. By Lemma 5 it suffices to prove that $T$ is $\left(1, P^{m}\right)$-summing. Write now $P^{\prime}$ for the (usual topological) dual of $P$. Since $P$ is finite dimensional, we have that the identity map is 1summing, and so for each finite family $h_{1}, \ldots, h_{l} \in P$

$$
\begin{aligned}
\sum_{i=1}^{l}\left\|T\left(h_{i}\right)\right\| & \leq\|T\| \sum_{i=1}^{l}\left\|h_{i}\right\|_{L^{p}(m)} \\
& \leq\|T\| K \sup _{y^{\prime} \in B_{P^{\prime}}} \sum_{i=1}^{l}\left|\left\langle h_{i}, y^{\prime}\right\rangle\right| \\
& =\|T\| K \sup _{\epsilon_{i}= \pm 1}\left\|\sum_{i=1}^{l} \epsilon_{i} h_{i}\right\|_{L^{p}(m)} \\
& \leq 4\|T\| K \sup _{\epsilon_{i}= \pm 1} \sup _{g \in B_{P^{m}}}\left\|\int \sum_{i=1}^{l} \epsilon_{i} h_{i} g d m\right\| \\
& \leq 4\|T\| K \sup _{g \in B_{R}} \sum_{i=1}^{n}\left\|\int h_{i} g d m\right\|,
\end{aligned}
$$

where $K$ is the 1 -summing norm of the identity map and the constant 4 comes from (19). Therefore, $T$ is $\left(1, P^{m}\right)$-summing and so $\left(q, P^{m}\right)$-summing for every $q \geq 1$.

When $m$ is a scalar measure then the spaces $L^{p}(m)$ and $L^{p^{\prime}}(m), 1<p<\infty$, are reflexive and hence their closed unit balls are weakly compact or, equivalently, $\tau_{m}$-compact. Besides, in this case $\left(q, L^{p^{\prime}}(m)\right)$-summability coincides with the usual absolute $q$-summability for operators. Therefore Theorem 9 can be considered an extension of the classical Dvoretzky-Rogers Theorem to spaces of integrable functions with respect to a vector measure.

Let us present some examples that show that all the requirements in (i) are needed for the result to be true. Recall that $X(\mu)$ is an order continuous Banach function space over a finite measure space $(\Omega, \Sigma, \mu)$.

\section{Remark 10.}

(1) $\tau_{m}\left(P^{m}\right)$-Sequential Compactness of $B_{P}$ Is a Necessary Requirement. Consider the vector measure $m: \Sigma \rightarrow X(\mu)$ given by $m(A):=\chi_{A}, A \in \Sigma$. In this case, $L^{1}(m)=X(\mu)$ and the integration map $I_{m}: L^{1}(m) \rightarrow X(\mu)$ is isomorphism. Take $P=L^{1}(m)$ that is not finite dimensional by assumption. The subspace $P^{m}$ of $L^{\infty}(m)$ generated by $\chi_{\Omega}$ is $m$-norming for $P$. Consider the $m$-bidual space $P^{m m}$ for $P$ defined as $P^{m m}=L^{1}(m)$. Obviously, $B_{P^{m}}$ is $\tau_{m}\left(P^{m m}\right)$-compact. Since the seminorm on $L^{1}(m)$ defined by $f \leadsto\left\|\int f \chi_{\Omega} d m\right\|=\|f\|$ coincides with the norm, we have that $P^{m}$ is $m$-norming for
$P$ but clearly $B_{P}$ is not $\tau_{m}\left(P^{m}\right)$-sequentially compact. Note that any other $m$-dual space for $P$ containing a function $g(w)>\delta$ for some $\delta>0$ satisfies the same property: $B_{P}$ is not compact for the topology $\tau_{m}\left(P^{m}\right)$. Observe also that the identity $L^{1}(m) \rightarrow L^{1}(m)$ is $\left(q, P^{m}\right)$-summing for each $1 \leq q<\infty$, since for each finite set $f_{1}, \ldots, f_{m} \in L^{1}(m)$,

$$
\sum_{i=1}^{n}\left\|f_{i}\right\|_{L^{1}(m)}^{q}=\sum_{i=1}^{n}\left\|\int f_{i} \chi_{\Omega} d m\right\|_{X(\mu)}^{q} .
$$

Note that the identity is $\tau_{m}\left(P^{m}\right)$ sequentially completely continuous trivially. This example shows clearly the difference between $q$-summing and $\left(q, P^{m}\right)$-summing operators. In the first case, Alaoglu's Theorem assures that the unit ball of the dual space is weak $^{*}$-compact, and this is enough to prove the Dvoretzky-Rogers theorem via Pietsch's Factorization Theorem. In the second case, the topological properties for the unit balls of the spaces involved must be given as additional requirements. This means that the corresponding summability property for the isomorphism does not assure our Dvoretzky-Rogers type theorem to hold.

(2) Not All the m-Dual Systems for a Finite Dimensional Space $P$ Satisfy the Requirements of Theorem 9. Consider again the vector measure given in the example given in (1). Take $P$ as the (finite dimensional) subspace of $L^{1}(m)$ generated by $\chi_{\Omega}$. First, take the $m$-dual system $P=P^{m}=P^{m m}$, with the understanding that $P$ and $P^{m m}$ are subspaces of $L^{1}(m)$ and $P^{m}$ is a subspace of $L^{\infty}(m)$. In this case, $B_{P}$ is $\tau_{m}\left(P^{m}\right)$-sequentially compact, $B_{P^{m}}$ is $\tau_{m}\left(P^{m m}\right)$-compact, and the identity map on $P$ that coincides with the integration operator is $\left(q, P^{m}\right)$-summable for each $1 \leq q<\infty$, providing all the requirements in (i) of Theorem 9.

However, take now $P^{m}=L^{\infty}(m)$ and $P^{m m}=L^{1}(m)$. Assume that the vector measure $m$ does not have relatively compact range. This happens, for example, when $X(\mu)=$ $L^{r}[0,1], 1 \leq r<\infty$ (see Example 3.61 in [6]). Then $B_{P}$ is $\tau_{m}\left(P^{m}\right)$-sequentially compact but $B_{P^{m}}=B_{L^{\infty}(m)}$ is not $\tau_{m}\left(P^{m m}\right)$-compact, since the topology $\tau_{m}$ induced on $L^{\infty}(m)=L^{\infty}(\mu)$ by $L^{1}(m)$ coincides with the topology of $X(\mu)$ on this space. To see this, just consider the seminorm

$$
L^{\infty}(m) \ni g \rightsquigarrow\left\|\int \chi_{\Omega} g d m\right\|=\|g\|_{X(\mu)} .
$$

Thus if $B_{P^{m}}$ is $\tau_{m}\left(P^{m m}\right)$-compact, this would imply compactness of $B_{L^{\infty}(m)}$ with respect to the topology of $X(\mu)$, and so it would imply that the range of the vector measure is relatively compact, since it is included in $B_{L^{\infty}(m)}$.

(3) The Topological Requirements for the m-Dual System Are Not Enough: The Assumption on the $\left(q, P_{m}\right)$-Summability of the Isomorphism Is Also Needed. Consider the vector measure $m$ defined as Lebesgue measure $\mu$ on $[0,1]$. Take any $1<$ $p<\infty$ and consider $P=L^{p}(\mu)$. Then we have that $P^{m}=$ $L^{p^{\prime}}(\mu)$ is $m$-dual for $L^{p}(\mu)$, and so the topology $\tau_{m}\left(L^{p^{\prime}}(\mu)\right)$ gives the weak topology for the reflexive space $L^{p}(m)$ (see Proposition 1). If we define the $m$-bidual $P^{m m}=L^{p}(m)$, we have that the topology $\tau_{m}\left(P^{m m}\right)$ for $P^{m}$ is given by the weak 
topology for $L^{p^{\prime}}[0,1]$. So both topological requirements in (i) of Theorem 9 are satisfied. Of course, no isomorphism from $P$ is $q$-summing for any $1 \leq q<\infty$, and so no isomorphism is $\left(q, P^{m}\right)$-summing, since in this case both definitions of summability coincide.

Example 11. The Vector Measure Associated with the Volterra Operator. Let $1 \leq r<\infty$ and let $\nu_{r}: \mathscr{B}([0,1]) \rightarrow L^{r}([0,1])$ be the Volterra measure, that is, the vector measure associated with the Volterra operator. This measure is defined as

$$
\nu_{r}(A)(t):=\int_{0}^{t} \chi_{A}(u) d u \in L^{r}([0,1]), \quad A \in \Sigma
$$

(see the explanation in [6, p. 113]; all the information about this measure can be found in different sections of [6]). It is known that the range of $\nu_{r}$ is relatively compact. This is a consequence of the compactness of the Volterra operator (see the comments after [6, Proposition 3.47]).

Let $1<p<\infty, m=v_{r}$, and consider a subspace $P$ of $L^{p}(m)=L^{p}\left(v_{r}\right)$. Assume that there is an $m$-dual space $P^{m}$ for $P$ such that $B_{P} \subseteq K B_{L^{\infty}(m)}$ for certain $K>0$ (e.g., a subspace generated by a finite set of functions in $L^{\infty}(m)$ with $L^{p}(m)$-norm greater than $\left.\delta>0\right)$. Take $P^{m m}$ as $L^{p}(m)$. Then $B_{P^{m}}$ is $\tau_{m}\left(P^{m m}\right)$-compact as a consequence of Theorem 10 in [18]. In this case, we have a simplified version of our Dvoretzky-Rogers type theorem for the subspace $P: P$ is finite dimensional if there is $1 \leq q<\infty$ such that the identity map is $\left(q, P^{m}\right)$-summing and $B_{P}$ is $\tau_{m}\left(P^{m}\right)$-sequentially compact.

\section{An Application: Subspaces of $L^{p}(m)$ That Are Fixed by the Integration Map}

In what follows we use our results in order to obtain information about subspaces of $L^{p}(m)$ spaces that are fixed by the integration map $I_{m}$. This topic has been studied since the very beginning of the investigations on the structure of the spaces of integrable functions with respect to a vector measure, and several papers on this topic have been published recently (mainly regarding subspaces that are isomorphic to $c_{0}$ and $\ell^{1}$, see [21] and the references therein). Let us show an easy example.

Example 12. Consider as in Remark 10 for $X(\mu)=L^{r}[0,1]$ the vector measure $m: \Sigma \rightarrow L^{r}[0,1]$ given by $m(A):=\chi_{A}$, $r \geq 1$. Consider the subspace $S$ generated by the Rademacher sequence in $L^{r}[0,1]$. By the Khintchine inequalities, $S$ is a subspace in $L^{r}[0,1]$ that is isomorphic to $\ell^{2}$. Recall that $L^{1}(m)=L^{r}[0,1]$ and the integration map is isomorphism. Obviously the restriction of the integral operator $I_{m}$ : $L^{1}(m) \rightarrow L^{r}[0,1]$ to $S$ is in fact the identity map. For $p \geq 1$ we have that $L^{p}(m)=L^{p r}[0,1]$, and again by the Khintchine inequalities $S$ is a subspace of $L^{p}(m)$ that is fixed by the integration map $I_{m}: L^{p}(m) \rightarrow L^{r}[0,1]$.

As we noted after the definition of $\left(q, L^{p^{\prime}}(m)\right)$-summing operator, the integration map from $L^{p}(m)$ for any $1 \leq$ $p<\infty$ is always $\left(q, L^{p^{\prime}}(m)\right)$-summing for every $q \geq 1$; in fact it is in a sense the canonical example of this kind of operators. Thus, our Dvoretzky-Rogers type result can be directly applied to obtain negative results on the existence of infinite dimensional subspaces of $L^{p}(m)$ that are fixed by $I_{m}$. We say that a subspace $P$ of $L^{P}(m)$ is fixed by the integration map if $\left.I_{m}\right|_{P}$ is isomorphism.

The following result shows that, under some compactness requirements, any subspace $S$ of $L^{p}(m)$ that is fixed by $I_{m}$ has to be finite dimensional. For the case in which the $m$ dual system that is considered is $P^{m}=L^{p^{\prime}}(m)$ and $P^{m m}=$ $L^{p}(m)$, conditions under which the balls of these spaces are $\tau_{m}$ compact are given in Corollary 8 of [18].

Corollary 13. Let $1 \leq p<\infty$, and let $P$ be a subspace of $L^{p}(m)$ that is fixed by the integration map. If there is an $m$-dual system for $P$ such that $B_{P}$ is $\tau_{m}\left(P^{m}\right)$-sequentially compact and $B_{P^{m}}$ is $\tau_{m}\left(P^{m m}\right)$-compact, then $P$ is finite dimensional.

Proof. It is a consequence of Theorem 9 and the fact that the integration map is $\left(q, L^{p^{\prime}}(m)\right)$-summing for every $1 \leq q \leq$ $\infty$.

In particular, the subspace $P$ generated by the Rademacher functions that has been shown in Example 12 does not have an $m$-dual system satisfying the compactness requirements in Corollary 13.

Remark 14. By [11, Theorem 3.6], if the vector measure $m$ has relatively compact range and $1<p<\infty$, then the restriction of the integration map to $L^{p}(m)$ is compact. Thus, if $S$ is a subspace of $L^{p}(m)$ that is fixed by the integration map, it has always finite dimension.

To finish, let us remark that as a consequence of the following result the ideas that prove Corollary 13 can be applied to maps acting in a subspace $P$ that is fixed by the integration map, other than the inclusion map.

Proposition 15. Let $1 \leq p<\infty$. Let $P$ be a subspace of $L^{p}(m)$ that is fixed by the integration map and let $P^{m} \subseteq L^{p^{\prime}}(m)$ be an $m$-dual space of $P$ containing $\chi_{\Omega}$. Then every operator $T$ : $P \rightarrow F$ with values on a Banach space $F$ is $\left(q, P^{m}\right)$-summable for every $1 \leq q<\infty$.

Proof. Let $T: P \rightarrow F$ be an operator with values on a Banach space $F$, and let $f_{1}, \ldots, f_{n} \in P$. Then

$$
\begin{aligned}
\sum_{i=1}^{n}\left\|T\left(f_{i}\right)\right\|^{q} \leq & \|T\|^{q} \cdot \sum_{i=1}^{n}\left\|f_{i}\right\|_{L^{p}(m)}^{q} \\
\leq & \|T\|^{q} \cdot\left\|\left(I_{m}\right)^{-1}\right\|^{q} \cdot \sum_{i=1}^{n}\left\|\int f_{n} d m\right\|^{q} \\
\leq & \|T\|^{q} \cdot\left\|\left(I_{m}\right)^{-1}\right\|^{q} \cdot\|m\|(\Omega)^{q / p} \\
& \cdot \sup _{g \in B_{p^{m}}} \sum_{i=1}^{n}\left\|\int f_{n} g d m\right\|^{q} .
\end{aligned}
$$

This gives the result. 


\section{Competing Interests}

The authors declare that they have no competing interests.

\section{Acknowledgments}

This work was supported by the Ministerio de Economía y Competitividad (Spain) under Grants MTM2015-66823-C22-P (P. Rueda) and MTM2012-36740-C02-02 (E. A. Sánchez Pérez).

\section{References}

[1] E. A. Sánchez Pérez, "Compactness arguments for spaces of pp-integrable functions with respect to a vector measure and factorization of operators through Lebesgue-Bochner spaces," Illinois Journal of Mathematics, vol. 45, no. 45, pp. 907-923, 2001.

[2] E. A. Sánchez Pérez, "Vector measure duality and tensor product representations of $L_{p}$-spaces of vector measures," Proceedings of the American Mathematical Society, vol. 132, no. 11, pp. 3319-3326, 2004.

[3] D. R. Lewis, "Integration with respect to vector measures," Pacific Journal of Mathematics, vol. 33, pp. 157-165, 1970.

[4] D. R. Lewis, "On integrability and summability in vector spaces," Illinois Journal of Mathematics, vol. 16, pp. 294-307, 1972.

[5] G. P. Curbera, "Banach space properties of $L^{1}$ of a vector measure," Proceedings of the American Mathematical Society, vol. 123, no. 12, pp. 3797-3806, 1995.

[6] S. Okada, W. J. Ricker, and E. A. Sánchez Pérez, Optimal Domain and Integral Extension of Operators Acting in Function Spaces, vol. 180 of Operator Theory: Advances and Applications, Birkhäuser, Basel, Switzerland, 2008.

[7] I. Ferrando, Duality in spaces of p-integrable functions with respect to a vector measure [Ph.D. thesis], Universidad Politécnica de Valencia, 2009.

[8] I. Ferrando, "Factorization theorem for 1-summing operators," Czechoslovak Mathematical Journal, vol. 61, no. 3, pp. 785-793, 2011.

[9] J. Lindenstrauss and L. Tzafriri, Classical Banach Spaces, II, Springer, Berlin, Germany, 1996.

[10] J. Diestel and J. J. Uhl, Vector Measures, vol. 15 of Mathematical Surveys and Monographs, American Mathematical Society, Providence, RI, USA, 1977.

[11] A. Fernández, F. Mayoral, F. Naranjo, C. Sáez, and E. A. Sánchez-Pérez, "Spaces of $p$-integrable functions with respect to a vector measure," Positivity, vol. 10, no. 1, pp. 1-16, 2006.

[12] S. Okada and W. J. Ricker, "The range of the integration map of a vector measure," Archiv der Mathematik, vol. 64, no. 6, pp. 512-522, 1995.

[13] S. Okada, W. J. Ricker, and L. Rodríguez-Piazza, "Compactness of the integration operator associated with a vector measure," Studia Mathematica, vol. 150, no. 2, pp. 133-149, 2002.

[14] S. Okada, W. J. Ricker, and L. Rodríguez-Piazza, "Operator ideal properties of vector measures with finite variation," Studia Mathematica, vol. 205, no. 3, pp. 215-249, 2011.

[15] I. Ferrando and E. A. Sánchez Pérez, "Tensor product representation of the (pre)dual of the $L^{p}$-space of a vector measure," Journal of the Australian Mathematical Society, vol. 87, no. 2, pp. 211-225, 2009.
[16] I. Ferrando and J. Rodríguez, "The weak topology on $L^{p}$ of a vector measure," Topology and its Applications, vol. 155, no. 13, pp. 1439-1444, 2008.

[17] F. Galaz-Fontes, "The dual space of $L^{p}$ of a vector measure," Positivity, vol. 14, no. 4, pp. 715-729, 2010.

[18] P. Rueda and E. A. Sánchez Pérez, "Compactness in spaces of p-integrable functions with respect to a vector measure," Topological Methods in Nonlinear Analysis, vol. 45, no. 2, pp. 641-653, 2015.

[19] P. Rueda and E. A. Sánchez-Pérez, "Factorization theorems for homogeneous maps on Banach function spaces and approximation of compact operators," Mediterranean Journal of Mathematics, vol. 12, no. 1, pp. 89-115, 2015.

[20] E. A. Sánchez Pérez, "Vector measure orthonormal functions and best approximation for the 4-norm," Archiv der Mathematik, vol. 80, no. 2, pp. 177-190, 2003.

[21] S. Okada, W. J. Ricker, and E. A. Sánchez Pérez, "Lattice copies of $c_{0}$ and $1^{\infty}$ in spaces of integrable functions for a vector measure," Dissertationes Mathematicae, vol. 500, pp. 1-68, 2014. 


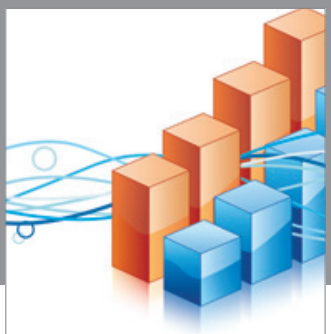

Advances in

Operations Research

vatem alat4

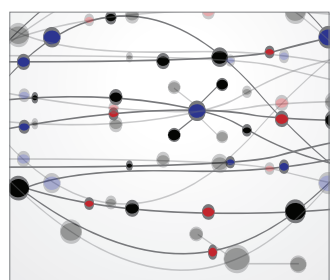

\section{The Scientific} World Journal
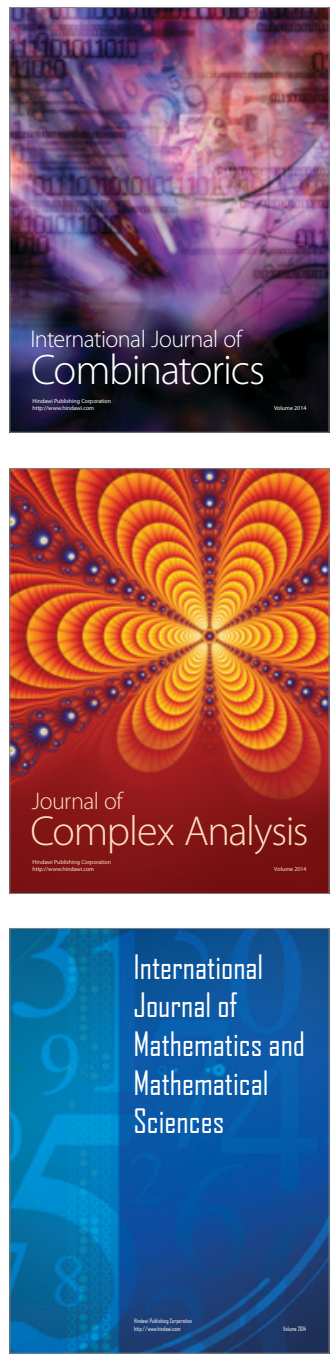
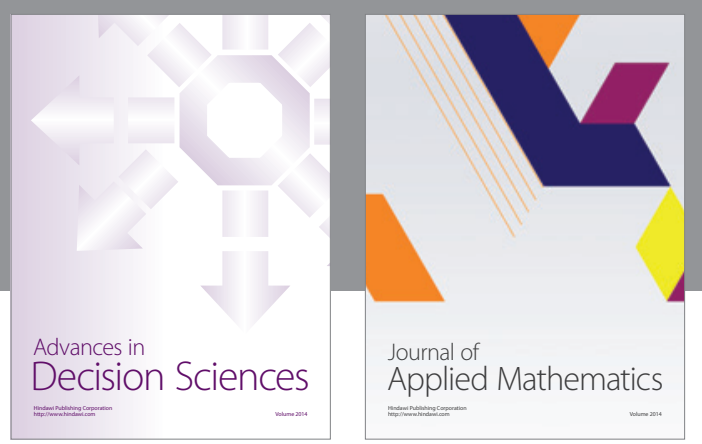

Algebra

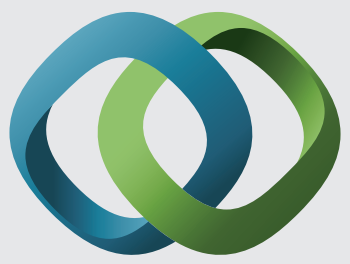

\section{Hindawi}

Submit your manuscripts at

http://www.hindawi.com
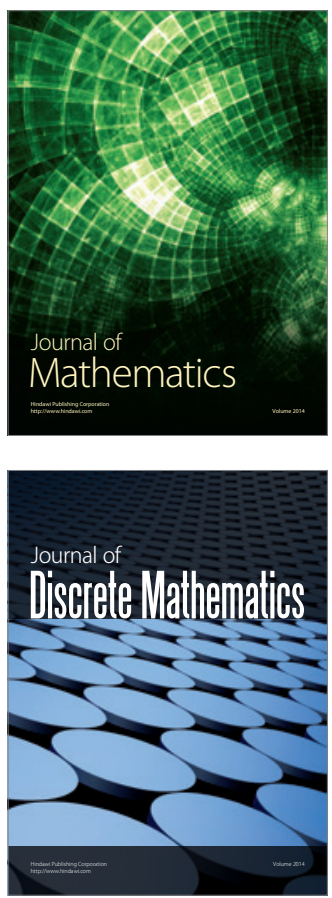

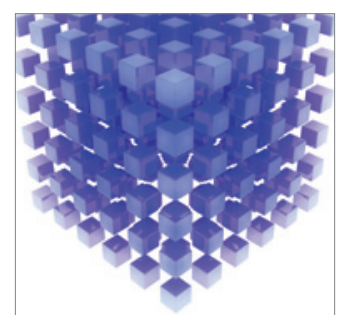

Mathematical Problems in Engineering
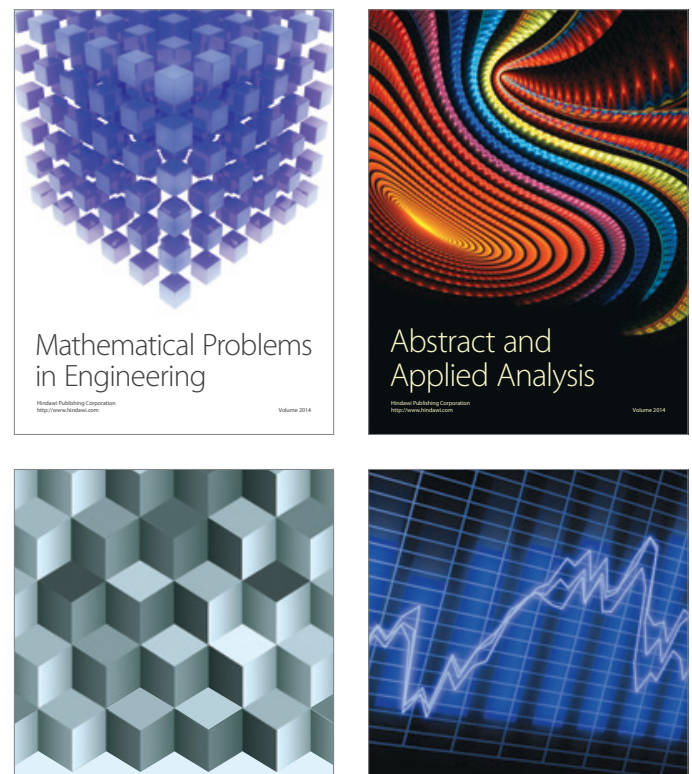

Journal of

Function Spaces

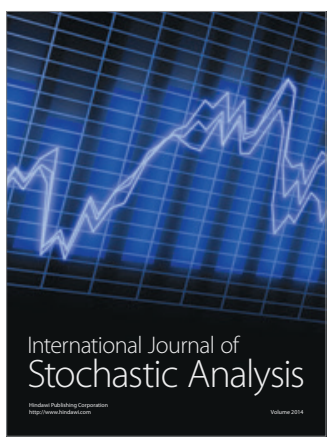

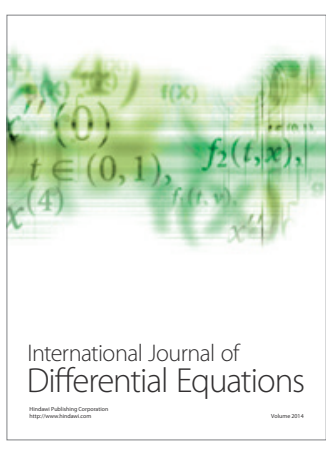
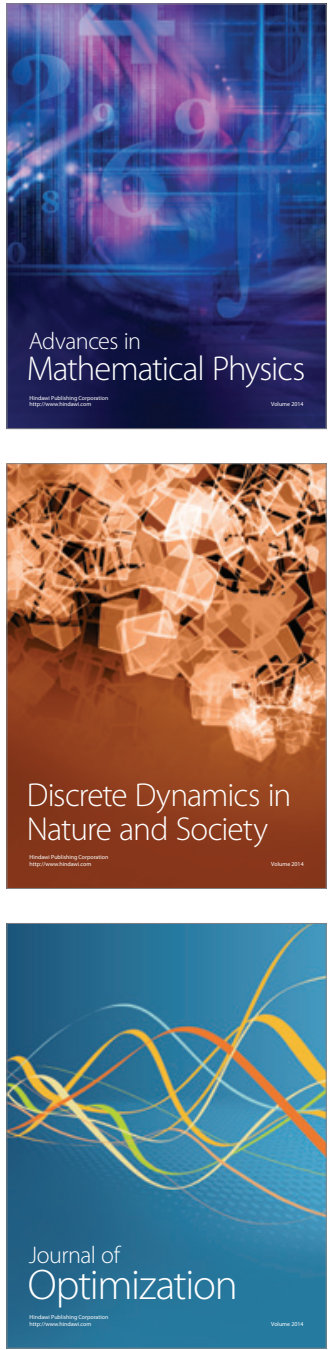